\title{
Electromagnetically induced transparency with a standing-wave drive in the frequency up-conversion regime
}

\author{
F. Silva \\ Departament d'Òptica, Universitat de València, E-46100 Burjassot, Spain
}

J. Mompart, V. Ahufinger, and R. Corbalán

Departament de Física, Universitat Autònoma de Barcelona, E-08193 Bellaterra, Spain

(Received 29 July 1999; revised manuscript received 9 February 2001; published 7 August 2001)

\begin{abstract}
We study electromagnetically induced transparency for a probe traveling-wave (TW) laser field in closed Doppler-broadened three-level systems driven by a standing-wave (SW) laser field of moderate intensity (its Rabi frequencies are smaller than the Doppler width of the driven transition). We show that probe windows of transparency occur for values of the probe to drive field frequency ratio $R$ close to half-integer values. For optical transitions and typical values of Doppler broadening for atoms in a vapor cell, we show that for $R>1$ a SW drive field is appreciably more efficient than a TW drive in inducing probe transparency. As examples, we consider parameters for real cascade schemes in barium atoms with $R \approx 1.5$ and in beryllium atoms with $R \approx 3.5$ showing that probe transmission values well above $50 \%$ are possible for conditions in which it is almost negligible either without driving field or with only one of the TW components of the drive. We show that a strongly asymmetric drive having two TW components with unequal intensities is even more eficient than a symmetric SW drive in inducing probe transparency. The case of arbitrary probe intensity is also considered.
\end{abstract}

DOI: 10.1103/PhysRevA.64.033802

PACS number(s): 42.50.Gy, 42.50.Hz, 32.80.Qk

\section{INTRODUCTION}

Linear and nonlinear optical properties of an atomic medium can be controlled and modified by applying a strong "drive" laser field that induces atomic coherence. Atomic coherence manifests itself in a rich variety of phenomena that are a subject of current research interest, such as electromagnetically induced transparency (EIT) (for reviews see, for instance, Refs. [1-4]); amplification and lasing without inversion (for a review, see Ref. [5]); high refractive index without absorption [6]; steep dispersion and either ultraslow group velocity [7] or gain-assisted superluminal light propagation [8]; and enhanced nonlinear optical susceptibility [9]. Here we consider EIT (in some cases it is also called "coherent population trapping" or "dark resonance" [2]), which is among the most basic of these phenomena and has many useful applications. For instance, it is at the heart of velocity selective coherent population trapping, a laser cooling method for free atoms which achieves subrecoil temperatures [10]. It is also at the basis of another very recent laser cooling method for trapped atoms which achieves efficient ground-state cooling [11]. EIT in a time-dependent regime has been used recently for stopping light [12], which could have important applications in the burgeoning field of quantum information.

In EIT, an otherwise absorbing medium is made transparent to a coherent field on resonance with a certain atomic transition by applying an intense coherent drive field to an adjacent transition. There has been a large number of theoretical contributions [1-4] and EIT was demonstrated experimentally in many systems [13-19].

Up to now, most theoretical papers dealing with atomic coherence effects in gas media have considered only the case of a traveling-wave (TW) drive field. In this paper we study
EIT in Doppler-broadened three-level media with standingwave (SW) drive (i.e., composed of two equal-frequency counterpropagating TW fields) and focus our analysis on the advantages of using a SW drive configuration instead of a TW one in the so-called frequency up-conversion regime where the probe wavelength is substantially shorter than that of the drive laser. A short account of some of the results presented below was published recently [20]. The possibility of inversionless amplification in the frequency up-conversion regime with an intense, detuned SW drive has been recently pointed out [21]. In the opposite regime of frequency downconversion, which appears in coherently pumped far-infrared lasers, it has been demonstrated both theoretically [22] and experimentally [23] that SW pumping of these lasers leads to gain values larger than those obtained for a TW pumping.

The case of a SW drive intuitively appears as promising in the frequency up-conversion regime. Let us consider, for instance, a Doppler-broadened three-level ladder scheme (Fig. 1) driven on transition $|2\rangle \leftrightarrow|3\rangle$ by a SW laser field and probed on transition $|1\rangle \leftrightarrow|3\rangle$ by a weak collinear TW laser field. The wave number and the detuning from atomic resonance are $\left(k_{d}, \Delta_{d}\right)$ and $\left(k_{p}, \Delta_{p}\right)$ for drive and probe fields, respectively. The resonance conditions for the processes contributing to probe absorption can be easily traced if the driveinduced ac Stark effect is neglected. Since the probe is weak, only processes involving one probe photon must be considered. Velocity-tuned multiphoton processes [24] involving one probe photon and several drive photons (see Fig. 2), with alternating absorption and emission from the two counterpropagating drive components, occur for velocities satisfying

$$
\begin{gathered}
\Delta_{p}-k_{p} v=q k_{d} v, \quad q=0, \pm 2, \ldots, \\
\Delta_{p}-k_{p} v=-\Delta_{d}+q k_{d} v, \quad q= \pm 1, \pm 3, \ldots,
\end{gathered}
$$




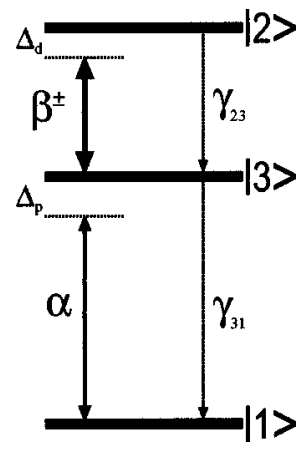

FIG. 1. Closed cascade three-level system under investigation.

where $v$ is the atomic velocity along the probe beam and $|q|$ is the number of drive photons involved in the process [25]. $q$ is positive (negative) if the drive photons absorbed in the multiphoton process are copropagating (counterpropagating) with the probe while those emitted are counterpropagating (copropagating). For a TW drive counterpropagating with the probe, only processes (1a) with $q=0$ and (1b) with $q$ $=-1$ occur. The resonance condition for this second process can be made $v$ - independent only if $k_{p}=k_{d}$. This is the well-known result that with TW fields Doppler-free absorption resonances only occur for equal-frequency drive and probe fields. In contrast, a SW drive allows also Doppler-free absorption resonances at $\Delta_{p}=0$ with $k_{p} / k_{d}=|q|=2,4, \ldots$, and at $\Delta_{p}+\Delta_{d}=0$ with $k_{p} / k_{d}=|q|=1,3, \ldots$, and it is therefore interesting in the frequency up-conversion regime.

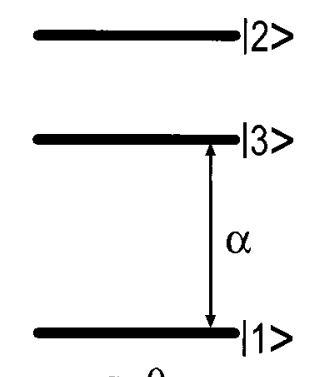

$\mathrm{q}=0$

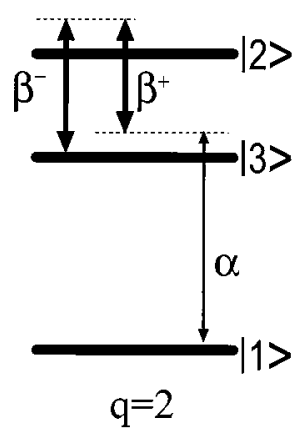

FIG. 2. Lowest-order processes participating in the probe absorption, with $q$ being the number of drive photons involved. Similar processes, interchanging $\beta^{+}$by $\beta^{-}$and $\beta^{-}$by $\beta^{+}$correspond to $q<0$.
(Note that there is also a Doppler-free absorption resonance at $\Delta_{p}=0$ with $k_{p} / k_{d}=q=0$; this case is relevant for coherently pumped long-wavelength far infrared lasers [22]). For the cascade scheme of Fig. 1 there will be a strong probe absorption under conditions of Doppler-free absorption resonances, since all the atoms, irrespective of their velocities, contribute to the absorption. Therefore, one expects to find windows of transparency for $k_{p} / k_{d} \simeq|q|+\frac{1}{2}$, i.e., approximately midway between consecutive Doppler-free multiphoton absorption resonances.

On the other hand, one must be aware that in a true SW drive (i.e., when its two TW components have the same intensity) there are nodes, where the total drive field vanishes. Therefore, the most abundant $v=0$ atoms placed at the nodes do not interact with the drive and will produce a strong resonant probe absorption. This undesirable feature from the perspective of EIT can in principle be avoided by using a drive field with unequal intensities for its two TW components. All these main conclusions will be confirmed by the detailed analysis presented below.

Section II is devoted to the semiclassical density matrix approach to the problem at hand. In Sec. III we briefly review the dressed-atom analysis for the SW excitation of a Doppler-broadened transition. Numerical results for EIT in two real cascade systems in $\mathrm{Ba}$ and in $\mathrm{Be}$ atoms are presented in Sec. IV. Section V summarizes our main conclusions.

\section{SEMICLASSICAL DENSITY MATRIX ANALYSIS}

To be specific, let us consider the closed cascade threelevel system shown in Fig. 1, although similar results can be obtained for the folded ( $V$ and $\Lambda$ ) schemes. The Hamiltonian $H_{0}$ that describes the internal energy of the three-level atoms is written as

$$
H_{0}=\sum_{i=1,2,3} E_{i}|i\rangle\langle i|
$$

and $\hbar \omega_{i j} \equiv E_{i}-E_{j}$ is the energy separation between levels $|i\rangle$ and $|j\rangle$. A TW laser field $E_{p}(z, t)=\frac{1}{2} A_{p} \exp \left[-i\left(\omega_{p} t-k_{p} z\right)\right]$ + c.c., to which transparency will be induced probes the lower transition $|3\rangle-|1\rangle$, while the upper transition $|2\rangle$ $-|3\rangle$ is driven by a collinear strong SW laser field $E_{d}(z, t)$ $=\sum_{\mu= \pm 1} \frac{1}{2} A_{d}^{\mu} \exp \left[-i\left(\omega_{d} t-\mu k_{d} z\right)\right]+$ c.c. The coupling of the atoms with the laser fields is characterized by the detunings $\Delta_{p}=\omega_{p}-\omega_{31}$ and $\Delta_{d}=\omega_{d}-\omega_{23}$ from their respective resonance, and by the three Rabi frequencies $2 \alpha \equiv \mu_{31} A_{p} / \hbar$ and $2 \beta^{ \pm} \equiv \mu_{23} A_{d}^{ \pm} / \hbar$, where $\mu_{31}$ and $\mu_{23}$ are electric dipole moments between the corresponding states. Without loss of generality the three Rabi frequencies will be taken to be real. Due to electric dipole selection rules the states $|2\rangle$ and $|1\rangle$ must have the same parity, therefore $\mu_{21}=0$. The Hamiltonian $V$ that describes the atom-laser coupling is given by

$$
\begin{aligned}
V= & -\sum_{\mu= \pm} \hbar \beta^{\mu} \exp \left[i\left(\omega_{d} t-\mu k_{d} z\right)\right]|3\rangle\langle 2|-\hbar \alpha \\
& \times \exp \left[i\left(\omega_{p} t-k_{p} z\right)\right]|1\rangle\langle 3|+\text { H.c. }
\end{aligned}
$$


where the standard rotating-wave approximation has been used to eliminate the nonresonant terms.

The three-level system is also subjected to relaxation processes, which will be described by means of a Liouville operator $R$ acting on the density matrix $\rho(v, z, t)$, whose evolution is given by

$$
i \hbar\left(\frac{\partial}{\partial t}+v \frac{\partial}{\partial z}\right) \rho=[H, \rho]+R \rho,
$$

where $H=H_{0}+V$, and $v$ is the atomic velocity along the $z$ axis. For simplicity, we consider that the relaxation operator $R$ only contains terms $\gamma_{31}$ and $\gamma_{23}$ describing the population decay rates of the middle and upper levels, respectively, and terms $\Gamma_{i j}$ describing the dephasing rates of the $\rho_{i j}$ offdiagonal elements. In the so-called radiative limit when there are no dephasing collisions one has $\Gamma_{21}=\Gamma_{12}=\gamma_{23} / 2 ; \Gamma_{32}$ $=\Gamma_{23}=\left(\gamma_{23}+\gamma_{31}\right) / 2$; and $\Gamma_{31}=\Gamma_{13}=\gamma_{31} / 2$.

It is convenient to work in the interaction picture to eliminate the fast dependences in the time evolution equations. Thus, we define new density matrix elements $\sigma_{i j}$ by

$$
\begin{aligned}
& \rho_{23}(v, z, t) \equiv \sigma_{23}(v, z, t) \exp \left(-i \omega_{d} t\right), \\
& \rho_{31}(v, z, t) \equiv \sigma_{31}(v, z, t) \exp \left(-i \omega_{p} t\right),
\end{aligned}
$$

and

$$
\rho_{21}(v, z, t) \equiv \sigma_{21}(v, z, t) \exp \left[-i\left(\omega_{d}+\omega_{p}\right) t\right] .
$$

The resulting equations of motion are

$$
\begin{aligned}
& \left(\frac{\partial}{\partial t}+v \frac{\partial}{\partial z}\right) \rho_{22}=-\gamma_{23} \rho_{22}+\left[i\left(\beta^{+} e^{i k_{d} z}+\beta^{-} e^{-i k_{d} z}\right) \sigma_{32}\right. \\
& \text { +c.c.], } \\
& \left(\frac{\partial}{\partial t}+v \frac{\partial}{\partial z}\right) \rho_{11}=\gamma_{31} \rho_{33}-\left(i \alpha e^{i k_{p} z} \sigma_{13}+\text { c.c. }\right) \text {, } \\
& \left(\frac{\partial}{\partial t}+v \frac{\partial}{\partial z}\right) \sigma_{23}=-R_{23} \sigma_{23}-i d_{23}\left(\beta^{+} e^{i k_{d} z}+\beta^{-} e^{-i k_{d} z}\right) \\
& -i \alpha e^{-i k_{p} z} \sigma_{21} \\
& \left(\frac{\partial}{\partial t}+v \frac{\partial}{\partial z}\right) \sigma_{31}=-R_{31} \sigma_{31}+i d_{13} \alpha e^{i k_{p} z} \\
& +i\left(\beta^{+} e^{-i k_{d} z}+\beta^{-} e^{i k_{d} z}\right) \sigma_{21}, \\
& \left(\frac{\partial}{\partial t}+v \frac{\partial}{\partial z}\right) \sigma_{21}=-R_{21} \sigma_{21}+i\left(\beta^{+} e^{i k_{d} z}+\beta^{-} e^{-i k_{d} z}\right) \sigma_{31} \\
& -i \alpha e^{i k_{p} z} \sigma_{23},
\end{aligned}
$$

where $d_{13}=\left(\rho_{11}-\rho_{33}\right), d_{23}=\left(\rho_{22}-\rho_{33}\right), R_{23}=\left(\Gamma_{23}-i \Delta_{d}\right)$, $R_{31}=\left(\Gamma_{31}-i \Delta_{p}\right)$, and $R_{21}=\left[\Gamma_{21}-i\left(\Delta_{p}+\Delta_{d}\right)\right]$. Since we are considering a closed system, the normalization condition $\rho_{11}+\rho_{22}+\rho_{33}=1$ must be added to Eqs. (5). Notice that there is an incoherent coupling between drive and probe fields through the population of level $|3\rangle$, and also a coherent coupling through the atomic coherence $\sigma_{21}$ [see Eqs. (5c) and (5d)]. All quantum interference phenomena mentioned in the introduction are based on this coherent coupling.

Even in the steady state, to which we restrict in the following, these equations do not have a general analytical solution due to the simultaneous presence in the right-hand sides of the explicit $z$ dependences $\exp \left( \pm i k_{d} z\right)$, obviously linked to the SW nature of the drive field. The solution must therefore be obtained numerically, in contrast with the wellknown case of a TW drive which can be treated analytically. In the case of weak probe and strong drive fields (i.e., $\alpha \ll \gamma_{31}, \beta^{ \pm} \gg \gamma_{23}$ ) one first solves Eqs. (5) to zeroth order in the probe field and to all orders in the drive field. This solution is well known from the theory of a high-intensity gas laser [26,27]: The density matrix elements are expanded in spatial Fourier components with a fundamental frequency $k_{d}$. One obtains a set of coupled difference equations in the Fourier coefficients $c_{n}$ which may be solved subject to appropriate boundary conditions on $c_{n}$ for large $n$. The solution can be expressed in terms of continued fractions [26,27]. This zeroth-order solution is then used to solve Eqs. (5) to first order in the probe field [28]. In some cases of practical interest it may be necessary to consider also a probe field of arbitrary intensity (e.g., in applications of EIT to enhance nonlinear processes the probe beam is not necessarily weak). The steady-state solution of Eqs. (5) for this case can be obtained introducing a two-index Fourier expansion for the density matrix elements with $k_{d}$ and $k_{p}$ fundamental spatial frequencies. However, this expansion can be more easily handled if we define, without loss of generality, two integers $a$ and $b$ such that $a / b$ is a rational number and $k_{d}=a k, k_{p}$ $=b k$, where $k$ is a reduced wave number [29]. Then the steady-state solution of Eqs. (5) becomes the one-index expansion:

$$
\begin{gathered}
\rho_{j j}=\sum_{q=-\infty}^{+\infty} y_{j j}^{q}(v) \exp (i q k z), \\
\sigma_{j k}=\sum_{q=-\infty}^{+\infty} y_{j k}^{q}(v) \exp (i q k z), \quad(j, k=1,2,3) .
\end{gathered}
$$

The set of coupled difference equations in the Fourier coefficients $y_{j j}^{q}$ and $y_{j k}^{q}$ can be recast in a form identical to that derived in Ref. [26] for complex numbers, but involving now vectors and matrices of dimension 4 [29]. The solution can therefore be obtained in terms of continued fractions of 4 $\times 4$ matrices [29]. The continued fractions are evaluated numerically by truncation to a finite number of terms in the denominator and it is a standard procedure in the calculations to check if the addition of one term in the denominator modifies the numerical value within the required accuracy. We have also checked that for $\alpha \ll \gamma_{31}$ the matrix continuedfraction solution reproduces the Feldman and Feld's solution [28].

The absorption and dispersion characteristics of the atomic medium with the ladder configuration shown in Fig. 1 are derived in the usual way from the components of the polarization $P(z, t)=\int N(v) d v\left[\mu_{23} \rho_{32}(v, z, t)\right.$ 
$\left.+\mu_{31} \rho_{13}(v, z, t)\right]+$ c.c., whose temporal and spatial oscillation matches the coupled field. Here $N(v) d v$ is the number of atoms per unit volume with velocity $v$ and we take for $N(v)$ the usual Maxwellian distribution with a most probable velocity $u$. Thus, for instance, the intensity absorption coefficient for the probe is given by $a_{p}$ $=\left(2 \hbar \omega_{p} \alpha / I_{p}\right) \int d v N(v) \operatorname{Im} y_{31}^{b}(v)$, where $I_{p}=\varepsilon_{0} c A_{p}^{2} / 2$ is the probe intensity.

We will consider here the following drive field configurations: (i) a symmetric $\mathrm{SW}$ drive (i.e., $\beta \equiv \beta^{+}=\beta^{-}$), (ii) a TW drive copropagating with the probe (i.e., $\beta \equiv \beta^{+}$and $\beta^{-}=0$ ), (iii) a counterpropagating TW drive (i.e., $\beta \equiv \beta^{-}$ and $\beta^{+}=0$ ), and (iv) an asymmetric SW drive (i.e., $\beta^{+}$ $\neq \beta^{-}$). In all cases the drive will be strong enough to saturate the driven transition, i.e., $\beta \gg \gamma_{23}$, although with a Rabi frequency smaller than the Doppler broadening $\Delta \omega_{D}$ of the driven transition, i.e., $\beta^{ \pm}<\Delta \omega_{D}=2 \sqrt{\ln 2}(u / c) \omega_{d}$. Let us emphasize that Doppler broadening is one of the main difficulties in observing and using atomic coherence effects in atomic gases, in particular in the case of a large frequency up-conversion ratio $R=\omega_{p} / \omega_{d}=k_{p} / k_{d}$. It is also well known that a way to mitigate the negative influence of Dop- pler broadening is to use a high-intensity TW drive with $\beta$ $\geqslant \Delta \omega_{D}$, which usually requires pulsed lasers. In contrast, we here explore the opposite regime, i.e., $\beta<\Delta \omega_{D}$, but use a SW drive.

\section{DRESSED-ATOM ANALYSIS}

The dressed-atom analysis [30] is very useful for the physical interpretation of numerical results obtained by solving the density matrix Eqs. (5) for the case of a weak probe field. Consider again the ladder scheme of Fig. 1, although generalization to any other three-level configuration is straightforward. We use a quantum description of the laser fields and denote by $\left|i ; N^{+}, N^{-} ; n_{p}\right\rangle$ the states of the uncoupled atom+laser fields, with the atom in state $i=1,2,3$, in the presence of $n_{p}$ probe photons and $N^{+}\left(N^{-}\right)$photons of the TW drive component copropagating (counterpropagating) with the probe field. For a TW drive it is either $N^{+}$ $=0$ or $N^{-}=0$. In the standard rotating-wave approximation, and considering only processes involving one probe photon, due to the weakness of the probe field, one has the following infinite chain of couplings (again due to the SW nature of the drive field) between the unperturbed states:

$$
\begin{aligned}
& \stackrel{\beta^{+}}{\cdots \leftrightarrow}\left|2 ; N^{+}, N^{-}-1 ; n_{p}\right\rangle \stackrel{\beta^{-}}{\leftrightarrow}\left|3 ; N^{+}, N^{-} ; n_{p}\right\rangle \stackrel{\beta^{+}}{\leftrightarrow}\left|2 ; N^{+}-1, N^{-} ; n_{p}\right\rangle \stackrel{\beta^{-}}{\leftrightarrow} \cdots \\
& \uparrow \alpha \\
& \left|1 ; N^{+}, N^{-} ; n_{p}+1\right\rangle \text {, }
\end{aligned}
$$

where we have indicated the matrix elements of the atomlaser interaction Hamiltonian between the levels connected by the arrows, in the semiclassical approximation. In this approximation, the choice of $N^{+}, N^{-}$, and $n_{p}$ is unrelevant, therefore we introduce the following shortened label:

$$
\begin{aligned}
\left|3 ; N^{+}-m, N^{-}+m ; n_{p}\right\rangle & \equiv|3 ; q=2 m, 0\rangle, \\
\left|2 ; N^{+}-m, N^{-}+m-1 ; n_{p}\right\rangle & \equiv|2 ; q=2 m-1,0\rangle, \\
\left|1 ; N^{+}, N^{-} ; n_{p}+1\right\rangle & \equiv|1 ; 0,1\rangle,
\end{aligned}
$$

with $m=0, \pm 1, \pm 2, \ldots$.

If the energy of the state $|3 ; 0,0\rangle$ is taken as the origin, the energies of the above unperturbed states become (with $\hbar$ $=1$ )

$$
\begin{gathered}
E_{3 ; q}^{u}=q k_{d} v, \quad q=\text { even, } \\
E_{2 ; q}^{u}=-\Delta_{d}+q k_{d} v, \quad q=\text { odd },
\end{gathered}
$$

$$
E_{\text {probe }}^{u}=\Delta_{p}-k_{p} v
$$

that represented as a function of $k_{d} v$ [see Fig. 3(a) for the case $\left.\Delta_{d}=0\right]$ appear as two manifolds of straight lines, one of them formed by lines with the even slope passing through the origin, and the other by lines with the odd slope passing through the point with coordinates $\left(0,-\Delta_{d}\right)$. The probe level is represented in Fig. 3(a) by a straight dotted-dashed line with ordinate at the origin $\Delta_{p}$ and slope $-k_{p} / k_{d}=-R$. Notice that the crossing points between the probe level and the other levels determine the (unperturbed) resonant velocities at which various multiphoton absorption resonances occur. Thus, the crossings of $E_{\text {probe }}^{u}$ with the levels of slopes $q=0,1,2$, and 3 determine the resonant velocities for the four processes shown in Fig. 2.

For a TW drive copropagating with the probe, only the following three states are involved: $|3 ; q=0,0\rangle$, $|2 ; q=1,0\rangle,|1 ; 0,1\rangle$. If the fields are counterpropagating the levels involved are $|3 ; q=0,0\rangle,|2 ; q=-1,0\rangle,|1 ; 0,1\rangle$.

The laser-atom coupling can be considered in two steps owing to the weak-probe assumption. First one considers the coupling with the strong SW drive field. By diagonalizing 
(a)

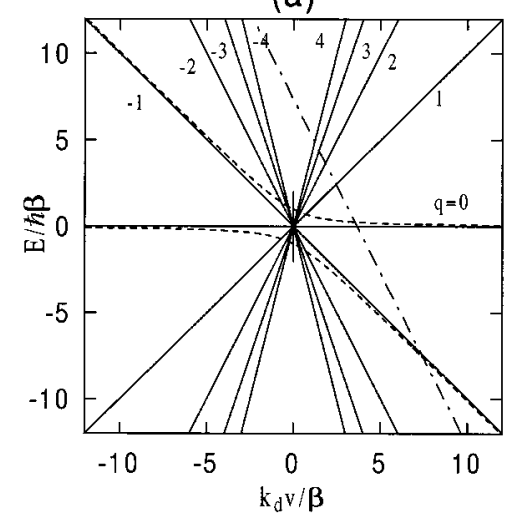

(b)

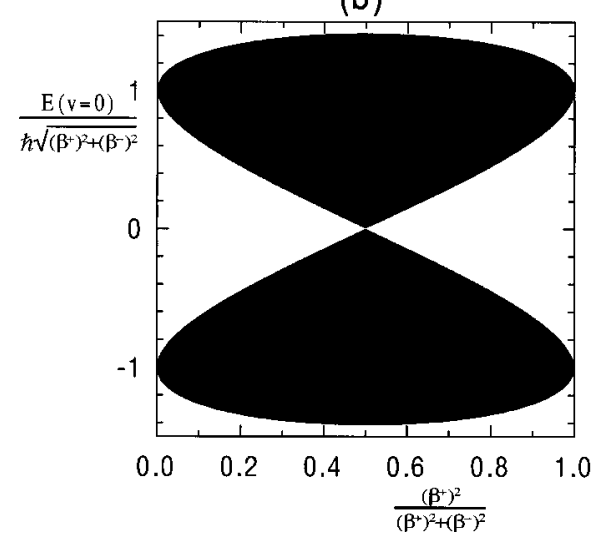

FIG. 3. (a) Energy diagram of the atom dressed by a resonat drive field (solid lines) with $\Delta_{d}=0$ and $\beta^{+}=\beta^{-}=\beta$. Dotted lines correspond to the case of a TW drive. The dotted-dashed line represents the probe level for $R=2.5, \Delta_{p}=10 \beta$. (b) The black domains represent the space-inhomogeneous continuous energy spectrum for $v=0$ atoms as a function of the asymmetry factor $\left[\left(\beta^{+}\right)^{2} /\left(\beta^{+}\right)^{2}\right.$ $\left.+\left(\beta^{-}\right)^{2}\right]$ of the drive field for a constant total intensity $\left(\beta^{+}\right)^{2}$ $+\left(\beta^{-}\right)^{2}$.

the infinite Hamiltonian associated with the first row in Eq. (7) one obtains the perturbed energies $E_{n}$ and eigenstates $\left|x_{n}\right\rangle$ of the so-called "SW-dressed two-level atom" [31]. The $\left|x_{n}\right\rangle$ states can be written in the form $\left|x_{n}\right\rangle$ $=\Sigma_{q^{\prime}=-\infty}^{+\infty} C_{q^{\prime}, n}\left|j ; q^{\prime}, 0\right\rangle$, with $j=3$ (2) for $q^{\prime}$ even (odd). These dressed states are labeled by the integer $-\infty<n<\infty$, whose choice is completely arbitrary. The coupling with the probe field is treated as a perturbation on the basis formed by the $\left|x_{n}\right\rangle$ states and the probe state $|1 ; 0,1\rangle$ and its coupling elements depend on the $|3 ; 0,0\rangle$ term in the expansion of $\left|x_{n}\right\rangle$. Within the weak-probe assumption the influence of the coupling with the probe on the energies $E_{n}$ and $E_{\text {probe }}^{u}$ is negligible. This coupling produces a transition rate $P_{|1 ; 0,1\rangle \rightarrow\left|x_{n}\right\rangle} \propto \alpha^{2}\left|C_{0, n}\right|^{2}$, with the probe absorption resonance occurring at the velocity where $E_{n}$ and $E_{\text {probe }}^{u}$ are degenerate.

The TW drive case is much simpler. One obtains the two perturbed energies $E_{n}$ and eigenstates $\left|x_{n}\right\rangle$ by diagonalizing the $2 \times 2$ matrix corresponding to the coupling of $|3 ; q=0,0\rangle$ and $|2 ; q=1,0\rangle$ (or of $|3 ; q=0,0\rangle$ and $|2 ; q=-1,0\rangle)$. The two $E_{n}$ are the branches of a hyperbola whose asymptotes are the unperturbed energy levels [see the dotted lines in Fig. 3(a)].
For $v \neq 0$, an exact solution of the SW-dressed two-level atom problem can be obtained analytically only when the drive is on resonance and $\beta^{+}=\beta^{-}=\beta$, while numerical treatment is required in other cases [31]. For $\Delta_{d}=0$ and $\beta^{+}=\beta^{-}=\beta$ [Fig. 3(a)], at $v \neq 0$ the energy levels are identical to the unperturbed ones, however, the associated eigenstates are linear combinations of the unperturbed states. The case $v=0$ can always be treated analytically. In fact, a stationary atom experiences the $\mathrm{SW}$ drive as a monochromatic field (which is not the case for moving atoms, due to the opposite Doppler shifts suffered by the two TW components of the drive). This case can therefore be described by the TW solution with a space-dependent Rabi frequency. For $\Delta_{d}=0$ and $\beta^{+} \neq \beta^{-}, v=0$ atoms at some places "see" both field components in phase and experience a maximum ac Stark splitting $2\left(\beta^{+}+\beta^{-}\right)$; in other places, where both fields have opposite phases, atoms experience a minimum ac Stark splitting $2\left|\beta^{+}-\beta^{-}\right|$; all intermediate splitting values correspond to atoms placed between maximum and minimum field positions. Thus, due to this inhomogeneous ac Stark splitting suffered by the stationary atoms, for a symmetric SW $\left(\beta^{+}\right.$ $=\beta^{-}$) drive energies at $v=0$ cover a finite interval between $-2 \beta$ and $2 \beta$ [Fig. 3(a)] [31]. For $\Delta_{d}=0$ and $\beta^{+} \neq \beta^{-}$, the black domains in Fig. $3(\mathrm{~b})$ represent the spatially inhomogeneous continuous energy spectrum of $v=0$ atoms as a function of the asymmetry factor $\left(\beta^{+}\right)^{2} /\left(\beta^{+}\right)^{2}+\left(\beta^{-}\right)^{2}$ for a constant total intensity $\left(\beta^{+}\right)^{2}+\left(\beta^{-}\right)^{2}$. Since $E(v=0)=0$ occurs only for a symmetric SW drive $\left(\beta^{+}=\beta^{-}\right)$stationary atoms contribute to resonant $\left(\Delta_{p}=0\right)$ probe absorption only in this case.

Notice that in the resonant and symmetric SW drive case [Fig. 3(a)], for $\Delta_{p}=0$ and $R=k_{p} / k_{d}=1,2,3, \ldots$, there is a complete superposition between the probe level and a dressed-state level, i.e., a complete cancellation of the Doppler effect, since all the atomic velocity classes contribute simultaneously to the probe absorption resonance.

\section{NUMERICAL RESULTS AND DISCUSSION}

For the numerical results shown in this section, we have considered parameters for two real cascade systems (Fig. 1) in barium and in beryllium atoms. Let us discuss first the case of barium driven by a resonant SW field with $\beta$ $=350 \mathrm{MHz}$ coupling the upper transition at $\lambda_{d}=821 \mathrm{~nm}$ and a weak field with $\alpha=0.035 \mathrm{MHz}$ probing the lower transition at $\lambda_{p}=554 \mathrm{~nm}$. Note that the probe-to-drive frequency ratio is in this case $R=1.48$ which, as pointed out in the introduction, makes this configuration very convenient to observe EIT with a SW drive. With $|1\rangle,|2\rangle$, and $|3\rangle$ being the levels ${ }^{1} S_{0}\left[m_{J}=0\right],{ }^{1} P_{1}\left[m_{J}=1\right]$, and ${ }^{1} D_{2}\left[m_{J}=2\right]$, respectively, and with all the laser fields circularly polarized with the same helicity, this three-level cascade system in ${ }^{138} \mathrm{Ba}$ is almost perfectly closed [32]. The spontaneous emission rates of lower and upper transitions read, respectively, $\gamma_{31}=19 \mathrm{MHz}$ and $\gamma_{23}=3.5 \mathrm{MHz}$. For a Maxwellian atomic velocity distribution corresponding to barium atoms in a vapor cell at $T=500 \mathrm{~K}$ one has $k_{d} u=930 \mathrm{MHz}$. For a resonant drive field $\left(\Delta_{d}=0\right)$, we plot in Fig. 4 a contour map of $N(v) \operatorname{Im} y_{31}^{b}(v)$ in the parameter plane $\Delta_{p}(v)=\Delta_{p}-k_{p} v$ 


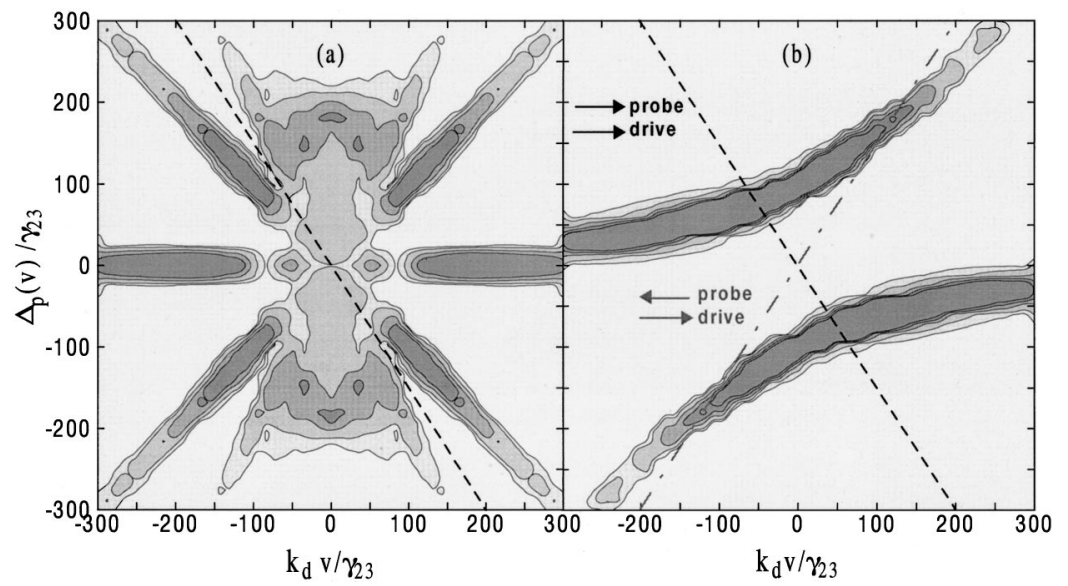

FIG. 4. Probe absorption contour map for $\mathrm{Ba}$ atoms in the parameter plane $\left[k_{d} v, \Delta_{p}(v)\right]$ for (a) resonant SW drive and (b) resonant TW drive. For parameters and discussion, see the text.
$=\Delta_{p}-R k_{d} v \quad$ vs $\quad k_{d} v$ for (a) a SW drive $\left(\beta^{+}=\beta^{-}\right.$ $=350 \mathrm{MHz})$ and (b) a copropagating TW drive $\left(\beta^{+}\right.$ $\left.=350 \mathrm{MHz}, \beta^{-}=0\right)$. Regions with dark gray (light gray) correspond to high (low) probe absorption. A comparison of Figs. 4(a) and 4(b) reveals that the absorption characteristics of the atomic medium are dramatically different for SW and TW driving. While for a TW drive [see Fig. 4(b)] the regions of high probe absorption occur close to the two branches of a hyperbola whose asymptotes have slopes 0 and 1 , for a SW drive high probe absorption occurs about straight lines passing through the origin with slopes $0, \pm 1, \pm 2, \ldots$ In Fig. 4(a) the regions with slopes 0 and \pm 1 are clearly visible, those with slopes \pm 2 can be appreciated clearly only far from the origin. Regions with higher slopes overlap and cannot be seen individually. These results clearly reflect the atom's dressed-level structure discussed in the previous section. To show this structure most clearly we have assumed in Fig. 4 a flat velocity distribution (i.e., $k_{d} u \rightarrow \infty$ ). The probe absorption domains in Fig. 4 are due to probe resonances involving state $|1 ; 0,1\rangle$ and the dressed states built by the drive field. The discussion in Sec. III then clarifies the physical origin of the probe absorption shown in Fig. 4(a) and shows that the main features in this figure are generic, i.e., with a resonant SW drive, probe absorption will always occur about straight lines through the origin, with the integer slope, in diagrams of the type shown in Fig. 4.

One can already "read" in Fig. 4 the advantages of using the resonant SW drive instead of the TW drive when $R>1$. To obtain the probe absorption coefficient $a_{p}$ one must perform an integration over the velocity distribution $N(v)$ in order to obtain the contributions of all the atoms. If, also, $\Delta_{p}=0$, the points representing different atomic velocity classes in the diagrams of Fig. 4 lie on a straight (dashed) line through the origin with slope $-R$ (or $R$ in the case of the counterpropagating probe and TW drive). To get $a_{p}$ one must add the contributions to probe absorption found along this dashed line. In Fig. 4(b), with $R>1$ there is always partial overlap between the dashed lines and the two absorbing regions, whose slope changes continuously from 0 to 1 . (Notice, however, that with counterpropagating drive and probe fields there is no absorption, i.e., complete transparency, for $0<R<1$, which corresponds to frequency downconversion.) For the SW drive case, we see in Fig. 4(a) that one gets small absorption not only when $0<R<1$ but also for $R \simeq n+1 / 2$ with $n=1,2,3 \ldots$ These features of the probe absorption coefficient are clearly seen in Fig. 5, where we plot the probe absorption coefficient normalized to its value in the absence of the drive field, $a_{p} / a_{p}(\beta=0)$, as a function of $R$ (and other parameters as mentioned at the beginning of this section) for both resonant SW drive and TW drive. Although Fig. 5 is not very interesting from a physical point of view, since one cannot explore in practice all the $R$ values with the same set of parameters (selected here for a cascade system in barium atoms with $R=1.48$ ), we plot it because it clearly exhibits the advantages of using resonant SW drive instead of TW drive in the frequency upconversion regime, i.e., when $R>1$. For SW drive we observe strong absorption resonances at integer values of the probe-to-drive frequency ratio, i.e., at $R=1,2,3 \ldots$ Between these absorption resonances there are probe windows of electromagnetically induced transparency at $R \simeq n+1 / 2$ $(n=1,2,3 \ldots)$ whose depth decreases as $R$ increases. It is also appreciated that up to $R=1$ a counterpropagating TW drive is the most convenient configuration in order to produce probe transparency. Nevertheless, for $R>1$ such that

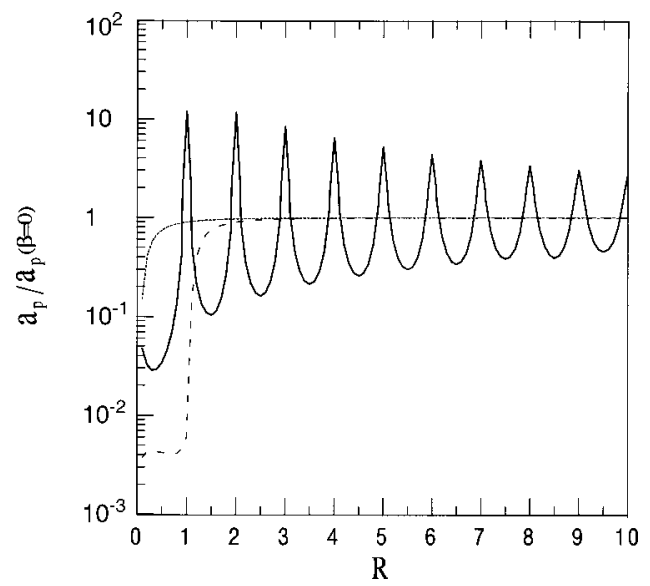

FIG. 5. Normalized probe absorption coefficient as a function of the probe-to-drive frequency ratio $R$ for $\Delta_{p}=\Delta_{d}=0$, and other parameters as in Fig. 4. Three different cases are represented: (i) SW drive (solid line), (ii) copropagating TW drive (dotted line), and (iii) counterpropagating TW drive (dashed line). 


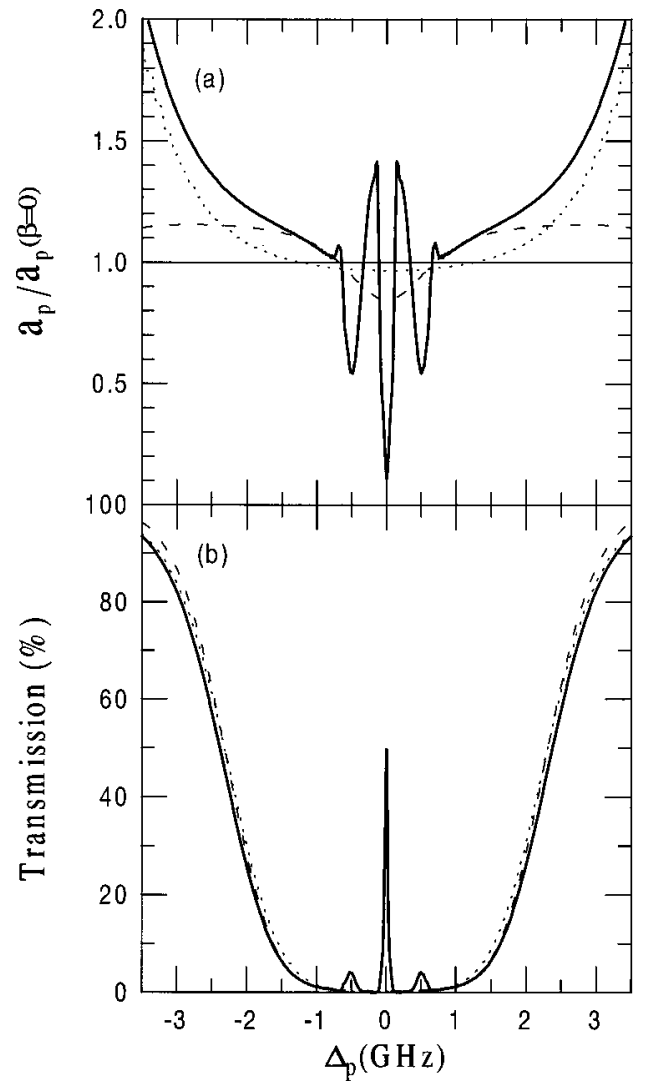

FIG. 6. (a) Normalized probe absorption coefficient as a function of the probe detuning $\Delta_{p}$ for (i) SW drive (solid line), (ii) copropagating TW drive (dotted line), and (iii) counterpropagating TW drive (dashed line). (b) Corresponding probe transmission through a vapor cell of barium atoms of length $L=4 \mathrm{~cm}$ and atomic density of $N=10^{15}$ atoms $/ \mathrm{cm}^{3}$. Other parameters as in Fig. 4.

$R \simeq n+1 / 2(n=1,2,3 \ldots)$, the resonant SW drive configuration is the most advantageous configuration to observe EIT or even the unique one in which it occurs, e.g., in Fig. 5 there is almost no EIT with a TW drive for $R \geqslant 2$ while there are appreciable EIT windows with a SW drive up to $R=9.5$.

Again, for the parameters of the real cascade system in atomic barium we have obtained the probe spectra shown in Fig. 6(a) for both SW and TW drive. Clearly, the maximum induced probe transparency is observed for a SW drive configuration and, as a characteristic feature of this case, three different peaks of transparency appear. In particular, at the probe field line center, i.e., $\Delta_{p}=0, a_{p}$ reduces to about $0.1 a_{p}(\beta=0)$ (i.e., it reduces by $\left.\sim 90 \%\right)$.

Finally, we have considered the probe propagation through a vapor cell of length $L=4 \mathrm{~cm}$ with an atomic density of $N=10^{15}$ atoms $/ \mathrm{cm}^{3}$. Assuming an electric dipole moment for the probed transition of $\mu \sim 10^{-27} \mathrm{C} \mathrm{cm}$ and neglecting drive field depletion through the vapor cell, we have obtained the probe transmission spectrum shown in Fig. 6(b). Probe transmission at the line center increases from the value $\exp (-10.6)$ in the absence of drive field to values above $50 \%$ with the SW drive, but remains almost negligible with a co- or a counterpropagating TW drive field. To obtain the same resonant probe transmission peak as in the SW case,

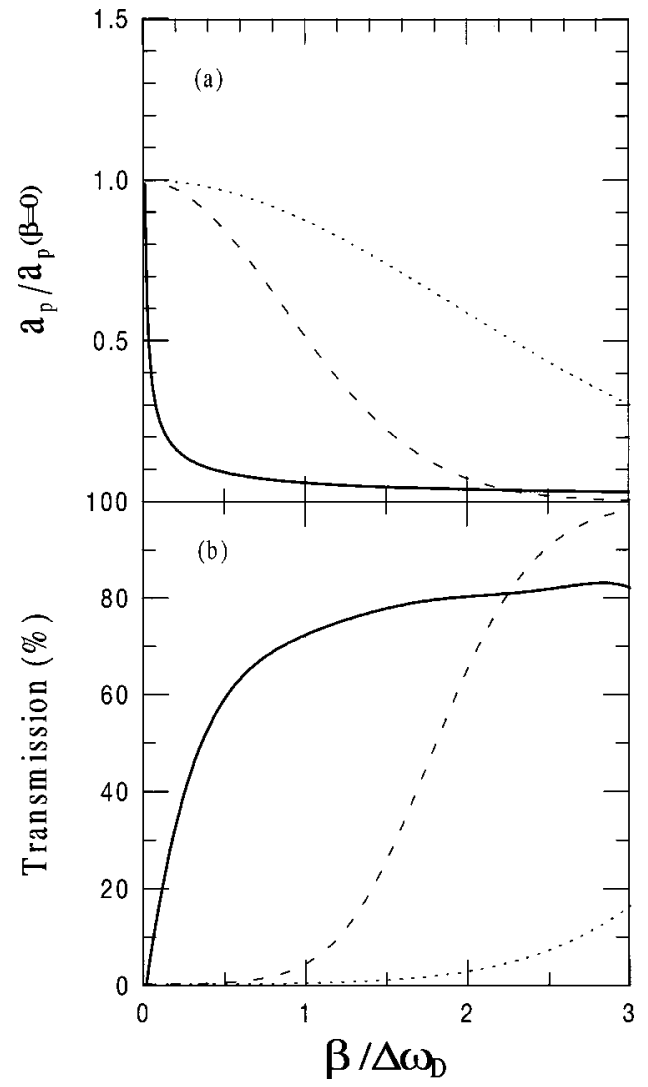

FIG. 7. Normalized probe absorption coefficient (a) and probe transmission (b) vs $\beta / \Delta \omega_{D}$ for $\Delta_{p}=0, \alpha=0.1 \gamma_{23}$, and other parameters as in Fig. 6 for (i) SW drive (solid line), (ii) copropagating TW drive (dotted line), and (iii) counterpropagating TW drive (dashed line).

with a counterpropagating TW drive one should use $\beta$ $\simeq 1680 \mathrm{MHz}$, which means an intensity 23 times that of one of the TW components of the SW drive used here. Additional information in this respect can be obtained from Fig. 7 where we plot, for $\Delta_{p}=0$ and $\alpha=0.1 \gamma_{23}$, the normalized probe absorption coefficient [Fig. 7(a)] and the probe transmission [Fig. 7(b)] versus $\beta / \Delta \omega_{D}$ and other parameters as in Fig. 6. One clearly sees that for $\beta / \Delta \omega_{D} \leqslant 2.2$ a SW drive produces larger probe transmissions than if only one of its TW components were used for driving. Above this value of $\beta / \Delta \omega_{D}$ a counterpropagating TW drive is more efficient than a SW one in inducing probe transparency.

We have computed also transmission spectra for increasing probe strength. The peak at $\Delta_{p}=0$ does not change appreciably for $\alpha$ up to $\alpha=3.5 \mathrm{MHz}=0.18 \gamma_{31}$. For $\alpha \sim 2 \gamma_{31}$ there is still a resonant peak above $50 \%$ but differences between the cases of TW and SW drive are less marked. Finally, we have computed transmission spectra for the same conditions as in Fig. 6 but for different drive detunings from atomic resonance. Results are qualitatively similar to those shown in Fig. 6(b) for drive detunings up to $\Delta_{d}$ $\approx 350 \mathrm{MHz}=\beta$, which indicates that it is not critical to tune the drive field exactly to resonance.

Up to now we have considered a drive field whose two components have the same intensity $\left(\beta^{+}=\beta^{-}\right)$. In spite of 


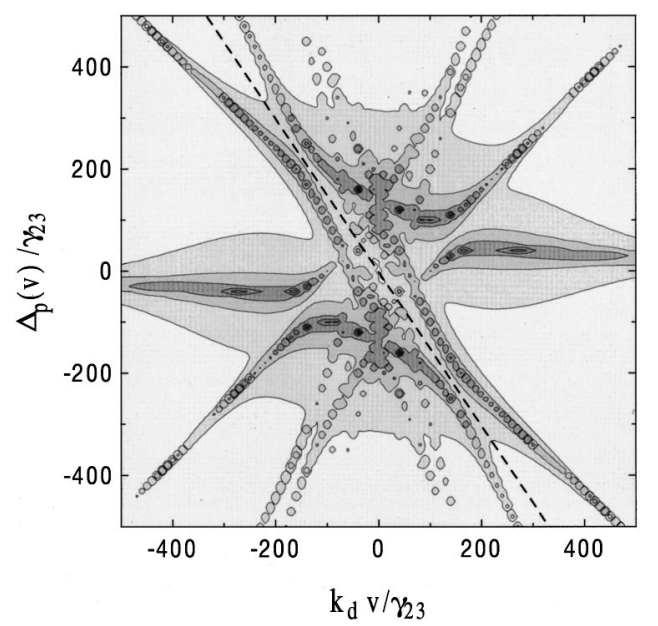

FIG. 8. Same as in Fig. 4(a) but for $\beta^{+}=383 \mathrm{MHz}$, $\beta^{-}=913 \mathrm{MHz}$.

the previous discussion, a SW field has a clear drawback for inducing transparency as compared to a TW field. In fact, while the latter field acts on all the atoms, the former does not couple to the most abundant $v=0$ atoms placed at the nodes of the SW where the drive field vanishes. These stationary atoms therefore contribute to probe absorption in the SW case. This drawback can be mitigated in part using a drive configuration with $\beta^{-}>\beta^{+}$. In fact, this case can be considered in a first approximation as consisting in a SW drive (convenient for $v \neq 0$ atoms) plus an extra counterpropagating TW drive (convenient for $v=0$ atoms). This is confirmed by the numerical results shown in Figs. 8 and 9. In Fig. 8 we represent a contour map of $N(v) \operatorname{Im} y_{31}^{b}(v)$ for $\beta^{+}=383 \mathrm{MHz}, \beta^{-}=913 \mathrm{MHz}$, and other parameters as for Fig. 4(a). The map is no more symmetric about $v=0$, and the dashed line representing different atomic velocity classes for $\Delta_{p}=0$ meets close to $v=0$ domains of smaller absorption than in Fig. 4(a). The resonant peak transmission as a

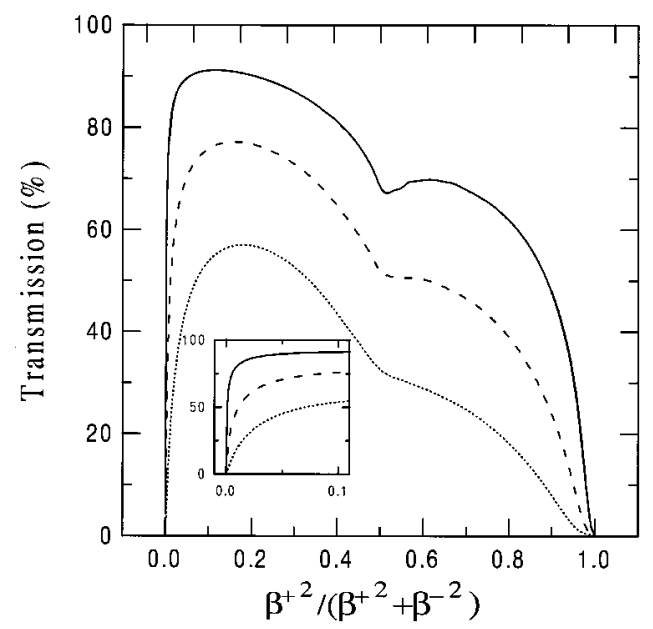

FIG. 9. Resonant peak transmission as a function of $\left(\beta^{+}\right)^{2} /\left[\left(\beta^{+}\right)^{2}+\left(\beta^{-}\right)^{2}\right]$ for several total drive intensities $\left(\beta^{+}\right)^{2}$ $+\left(\beta^{-}\right)^{2}$ and other parameters as for Fig. 6(b). From top to bottom curves correspond to $\left[\left(\beta^{+}\right)^{2}+\left(\beta^{-}\right)^{2}\right] / \gamma_{23}^{2}=8 \times 10^{4}, 2 \times 10^{4}$, and $5 \times 10^{3}$.

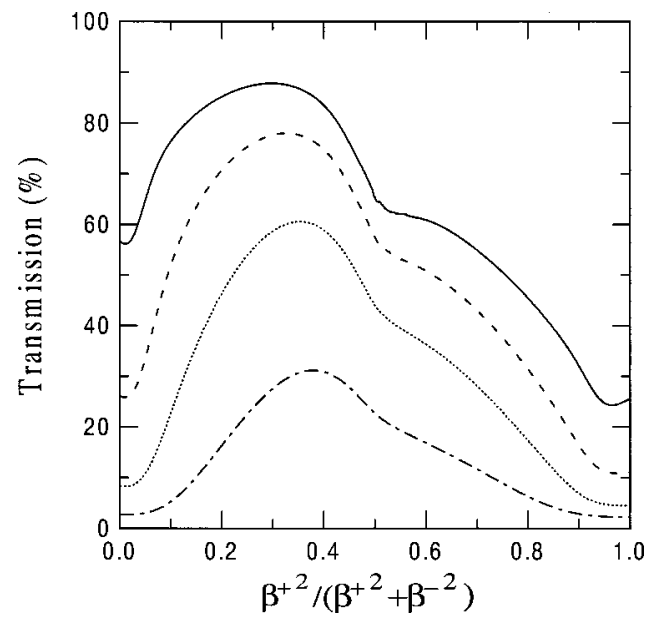

FIG. 10. The same as in Fig. 9 but now for Be atoms. From top to bottom curves correspond to $\left[\left(\beta^{+}\right)^{2}+\left(\beta^{-}\right)^{2}\right] / \gamma_{23}^{2}=12.6 \times 10^{4}$, $7 \times 10^{4}, 3 \times 10^{4}$, and $8 \times 10^{3}$.

function of $\left(\beta^{+}\right)^{2} /\left[\left(\beta^{+}\right)^{2}+\left(\beta^{-}\right)^{2}\right]$ for several total drive intensities $\left(\beta^{+}\right)^{2}+\left(\beta^{-}\right)^{2}$ and other parameters as for Fig. $6(\mathrm{~b})$ is plotted in Fig. 9. For each fixed total drive intensity there is a different optimum value of the asymmetry between $\beta^{-}$and $\beta^{+}$at which probe transmission is maximum.

Inspection of atomic data tables reveals that there are many probe transitions involving the ground state and having an adjacent transition to be driven such that $R \approx n+1 / 2$ ( $n$ $=1,2,3, \ldots)$. Examples include $\mathrm{Ba}(R=1.48), \mathrm{Cu}(R$ $=2.49), \mathrm{Be}(R=3.51)$, and $\mathrm{Hg}(R=5.48)$. To show that the advantages of using in EIT a SW drive instead of a TW one does not restrict to the particular case of barium atoms, we discuss briefly next the case of beryllium. Neglecting hyperfine structure and using drive and probe fields linearly polarized along the quantization axis, the levels $2 s{ }^{1} S_{0}, 2 p{ }^{1} P_{1}$, and $3 s^{1} S_{0}$ in beryllium form a cascade three-level system with $\lambda_{p}=234.86 \mathrm{~nm}, \lambda_{d}=825.4 \mathrm{~nm}$ (i.e., $R=3.51$ ), $\gamma_{31}$ $=85.8 \mathrm{MHz}$, and $\gamma_{23}=6.06 \mathrm{MHz}$. Taking other parameters as for $\mathrm{Ba}$, we plot in Fig. 10 transmission curves for $\mathrm{Be}$ analogues to those in Fig. 8. In both cases peak transmissions above $85 \%$ are possible with a strongly asymmetric drive.

\section{CONCLUSIONS}

In this paper we have analyzed, in the framework of standard semiclassical density matrix formalism, EIT for a probe TW field in closed Doppler-broadened three-level systems driven by a SW field. Electromagnetically induced probe transparency windows appear close to half integer values of the probe to drive field frequency ratio $R$. These results have been compared to those obtained for a TW drive proving that for $R>1$ a SW drive field is appreciably more efficient than a TW drive in inducing probe transparency. In particular, we have considered parameters for a real closed cascade scheme in barium atoms with $R \simeq 1.5$ showing probe transmission resonances with values above $50 \%$ when the transmission is almost negligible either without drive field or with only one of the TW components of the SW drive. We have shown that a drive field with two counterpropagating components of dif- 
ferent intensity is even more efficient than a SW one in inducing probe transparency. In ladder schemes, to achieve optimum probe transmission with a given total drive intensity, the drive component counterpropagating with the probe should be stronger. Many real three-level systems exist with $R \approx n+1 / 2(n=1,2,3, \ldots)$. In addition to the Ba case, another system in beryllium atoms with $R=3.51$ has been considered explicitly.

Detailed contour maps of the probe field absorption in the parameter plane $\left[k_{d} v, \Delta_{p}(v)\right]$ have been presented and discussed with the help of the dressed-atom approach.

\section{ACKNOWLEDGMENTS}

Support from the DGESIC (Spanish Government) under Contract No. PB98-0935-C03 and from the DGR (Catalan Government) under Contract No. 1999SGR00096 is acknowledged.
[1] M. O. Scully and M. S. Zubairy, Quantum Optics (Cambridge University Press, Cambridge, U.K., 1997), Chap. 7, pp. 220245.

[2] E. Arimondo, in Progress in Optics $X X X V$, edited by E. Wolf (Elsevier, Amsterdam, 1996), Chap. V, pp. 258-354.

[3] S. E. Harris, Phys. Today 50, 36 (1997).

[4] J. P. Marangos, J. Mod. Opt. 45, 471 (1998).

[5] J. Mompart and R. Corbalán, J. Opt. B: Quantum Semiclassical Opt. 2, R7 (2000).

[6] M. O. Scully, Phys. Rev. Lett. 67, 1855 (1991); A. S. Zibrov et al., ibid. 76, 3935 (1996).

[7] L. V. Hau, S. E. Harris, Z. Dutton, and C. H. Behroozi, Nature (London) 397, 594 (1999); M. M. Kash, V. A. Sautenkov, A. S. Zibrov, L. Hollberg, G. R. Welch, M. D. Lukin, Y. Rostovtsev, E. S. Fry, and M. O. Scully, Phys. Rev. Lett. 82, 5229 (1999); D. Budker, D. F. Kimball, S. M. Rochester, and V. V. Yashchuk, ibid. 83, 1767 (1999); S. Inouye, R. F. Löw, S. Gupta, T. Pfau, A. Görlitz, T. L. Gustavson, D. E. Pritchard, and W. Keterlee, ibid. 85, 4225 (2000).

[8] L. J. Wang, A. Kuzmich, and A. Dogariu, Nature (London) 406, 277 (2000).

[9] K. Hakuta, L. Marmet, and B. P. Stoichef, Phys. Rev. Lett. 66, 596 (1991); G. Z. Zhang, K. Hakuta, and B. P. Stoichef, ibid. 71, 3099 (1993); Y. Li and M. Xiao, Opt. Lett. 21, 1064 (1996).

[10] A. Aspect et al., Phys. Rev. Lett. 61, 826 (1988).

[11] G. Morigi, J. Eschner, and C. H. Keitel, Phys. Rev. Lett. 85, 4458 (2000); C. F. Roos, D. Leibfried, A. Mundt, F. SchmidtKaler, J. Eschner, and R. Blatt, ibid. 85, 5547 (2000).

[12] Ch. Liu, Z. Dutton, C. H. Behroozi, and L. V. Hau, Nature (London) 409, 490 (2001); D. F. Phillips, A. Fleischhauer, A. Mair, R. L. Walsworth, and M. D. Lukin, Phys. Rev. Lett. 86, 783 (2001).

[13] G. Alzetta, A. Gozzini, L. Moi, and G. Orriols, Lett. Nuovo Cimento Soc. Ital. Fis. 17, 333 (1976).

[14] H. R. Gray, R. M. Whitley, and C. R. Stroud, Jr., Opt. Lett. 3, 218 (1978).

[15] K.-J. Boller, A. Imamoğlu, and S. E. Harris, Phys. Rev. Lett. 66, 2593 (1991); J. E. Field, K. H. Hahn, and S. E. Harris, ibid. 67, 3062 (1991).
[16] K. Hakuta, L. Marmet, and B. Stoicheff, Phys. Rev. Lett. 66, 596 (1991).

[17] M. Xiao, Y.-q. Li, S.-z. Jin, and J. Gea-Banacloche, Phys. Rev. Lett. 74, 666 (1995).

[18] J. R. Boon, E. Zekou, D. J. Fulton, and M. H. Dunn, Phys. Rev. A 57, 1323 (1998).

[19] S. Wielandy and A. L. Gaeta, Phys. Rev. A 58, 2500 (1998).

[20] F. Silva, J. Mompart, V. Ahufinger, and R. Corbalán, Europhys. Lett. 51, 286 (2000).

[21] F. Silva, V. Ahufinger, J. Mompart, and R. Corbalán, Laser Phys. 9, 858 (1999).

[22] F. Silva, R. Corbalán, and R. Vilaseca, Opt. Commun. 114, 519 (1995).

[23] R. Corbalán, A. N. Pisarchik, V. N. Chizhevsky, and R. Vilaseca, Opt. Commun. 133, 225 (1997).

[24] J. Reid and T. Oka, Phys. Rev. Lett. 38, 67 (1977).

[25] For any of the processes of the type shown in Fig. 2 there is a family of processes obtained by the addition to any of the original $\alpha, \beta^{+}$, or $\beta^{-}$steps of an arbitrary even number of $\beta^{+}$ and/or $\beta^{-}$absorption-emission steps. These processes do not produce new atomic resonances but result in a modification of the resonance conditions given in Eq. (1) due to the driveinduced ac Stark effect, which is fully taken into account in the rest of the paper.

[26] See, e.g., S. Stenholm and W. E. Lamb, Jr., Phys. Rev. 181, 618 (1969).

[27] B. J. Feldman and M. S. Feld, Phys. Rev. A 1, 1375 (1970).

[28] See, e.g., B. J. Feldman and M. S. Feld, Phys. Rev. A 5, 899 (1972).

[29] R. Vilaseca, G. Orriols, L. Roso, R. Corbalán, and E. Arimondo, Appl. Phys. B: Photophys. Laser Chem. B34, 73 (1984).

[30] C. Cohen-Tannoudji, J. Dupont-Roc, and G. Grynberg, AtomPhoton Interactions (Wiley, New York, 1992).

[31] L. Roso, R. Corbalán, G. Orriols, R. Vilaseca, and E. Arimondo, Appl. Phys. B: Photophys. Laser Chem. B31, 115 (1983).

[32] P. B. Sellin, G. A. Wilson, K. K. Meduri, and T. W. Mossberg, Phys. Rev. A 54, 2402 (1996). 